\title{
PROBIOTICS AND HEALTH: A NEW EMERGING MODALITY IN BIO-THERAPEUTICS
}

Cortizo F. Probiotics and Health: A new emerging modality in Bio-therapeutics, Annal Dent Univ Malaya 1999; 6: 47 51 .

\section{INTRODUCTION: What are probiotics?}

The word "pro-biotics" derives from two Greek words meaning for life and refers to organisms that are incorporated into foods or supplements with the aim of reestablishing a healthy intestinal flora. A more recent definition of the term states: "a mono or mixed" culture of live micro-organisms which, applied to man or animal, affects beneficially the host animal by improving intestinal microbial balance"(1). The micro-organisms commonly used as probiotics for humans are the lactic acid bacteria (LAB)

Our general health status depends to a large extent upon the presence, number and relationship of the normal friendly bacteria of the gastrointestinal (GI) tract.

The recognised ability of the intestinal microflora and probiotic micro-organisms to exert a wide number of beneficial and potentially therapeutic effects, without the side effects normally encountered by traditional drug therapies, has created a great deal of interest in the use of probiotic bacteria as a potential bio-therapeutic.

\section{THE DIGESTIVE TRACT AND THE FRIENDLY ORGANISMS}

Over 100 types of bacteria inhabit our digestive tract altogether weighing approximately 1.5 to $2.0 \mathrm{~kg}$. Some of these organisms are permanent residents, while others are transient in nature. These organisms could either be anaerobic or aerobic bacteria, or both. Within a few days of birth the GI tract is colonised by bacteria that enter the system through the mouth.

The pattern of birth and type of feeding strongly influence the type of bacteria which establishes, either as transient or permanent, in the intestinal system. During the first two days of life, the large intestine of neonates fed with breast milk and supplementary cow's milk is colonised by enterobacteriaceae, streptococci including enterococci and clostridia(2). By day seven bifidobacteria become predominant $\left(10^{11}\right.$ organisms per gram of faeces) while the other groups decrease significantly in number. It is interesting to note that a dramatic shift has been observed in the species and strains of colonic microflora found in breast fed infants worldwide.

It has been suggested that a possible explanation for the change in the colonisation patterns may be a consequence of increasing contamination of the human environment including, air, water and foods (antibiotics, chemicals, petrol, pesticides, toxic substances, etc.) coupled with a decline in numbers of strains of bifidobacteria in breast fed babies and a rise in the levels of undesirable pathogenic organisms.
Fernando Cortizo, B. Sc. (Honours), Ph.D.

OMX Marketing Australia P/L

Melbourne

Australia

\section{FACTORS AFFECTING THE HUMAN COLONIC} MICROFLORA

Bacteria represent an integral part of the intestinal system throughout the lifetime of a person. The bacteria, which colonise our bodies, may be either good or harmful. Under ideal conditions of health and diet, a delicate and crucial balance is maintained between good and bad bacteria in the intestinal system. The maintenance of this internal balance is necessary to sustain good health and to support the normal processes of the body. However, this delicate balance is disrupted or influenced by three major factors:

\section{i) Host factors}

The host factors are particular to each individual and include, among others, acid $(\mathrm{HCl}$, lactic acid and fatty acids) enzymes (gastric and pancreatic) and bile salts production. As well as peristalsis activity, villous contraction, epithelial turnover and redox potential.

\section{ii) Microbial factors}

The Microbial factors include bacterial interaction whether antagonistic, symbiotic or synergistic and the traits of the individual organisms involved in relation to their growth requirements, adhesion properties and most importantly production of organic acids and of natural antibiotics. The production of high levels of organic acids is the most significant trait, which ensures their effectiveness in competing with other pathogenic organisms.

\section{iii) Environmental factors}

The environmental factors are, perhaps, the most significant and include widespread and excessive use of antibiotics either by prescription or its extensive application in agriculture, stockfeed and animal farming techniques. Another important factor is the dramatic changes in our diet from the traditional homemade meals to the high-sugar, junk food of modern day. These highly processed foods provide little or no nutritional value and encourage the growth of harmful bacteria. Of further concern is the chemical contamination of our food, especially meat and other animal products, which are often laced, with residues of hormones and antibiotics. Last but not least is modern technology and environmental hazards, which include household, workplace and other environmental chemicals. 
AREA OF APPLICATION OF PROBIOTICS FOR HUMANS: Established and postulated functions of colonic microflora

The intestinal flora is made up of different bacterial species, and thus able to produce a variety of enzymes and metabolites which perform the various types of metabolism in the intestine and influence the host's health and resistance to diseases. Within the intestine, the bacteria are implicated in the conversion of various substances that produce beneficial products to the host. In addition, bacterial cell components produced by some bacterial species modify the host's immune response by enhancing immune function.

The beneficial intestinal microflora protects the intestinal tract from proliferation or infection by harmful bacteria. The microflora of the intestinal system has the potential to be of significant benefit in various areas of human health. A summary of these benefits is listed below(3):

- Improvement in lactose intolerance

- Control and reduction in blood cholesterol levels

- Manufacture of vitamins and co-factors such as biotin, B3, B6, and folic acid.

- Enhancement of resistance against pathogens by producing natural organic acids such as lactic, acetic and formic acids which strongly inhibit harmful bacteria and microorganisms which are incapable of thriving in an acidic environment.

- Enhancement of resistance against pathogens by producing natural antibiotics substances such as acidophilin, lactolin and acidolin .

- significant anti-tumour activity

- Reduction in the risk of colon cancer

- Reduction in levels of faecal enzymes associated with colon cancer

- Prevention or reduction of constipation

- Prevention and treatment of diarrhoea

- Reduction and prevention of irritable bowel syndrome

- Detoxification of chemicals and carcinogenic substances

These benefits can be summarised in four basic functions accomplished by the intestinal microflora and probiotics:

\section{a) Metabolic function:}

Metabolic and Biochemical effects of the probiotic bacteria include metabolism of carbohydrates, proteins, bile acids, cholesterol and organic acids.

It has been reported that some strains of lactic acid bacteria can assimilate cholesterol(4) and deconjugate bile acids(5) in this way able to reduce cholesterol levels. It has also been suggested that probiotics, which produce propionic acid, could reduce the synthesis of cholesterol.

Probiotics have also been shown to assist in the reduction in the symptoms associated with lactose intolerance by producing beta-galactosidase which degrades lactose before it reaches the lower part of the small intestine ${ }^{6}$. The colon is the major site of bacterial colonisation, which salvage energy through the metabolism of undigested carbohydrates and gut secretions. The major products of carbohydrate metabolism are the short chain fatty acids (SCFA), acetate, lactate, propionate and butyrate(7). These SCFA in addition to lowering the $\mathrm{pH}$, appear to promote the flow of blood through the colonic vasculature while propionate enhances muscular activity and epithelial cell proliferation. Butyrate appears to promote a normal cell phenotype as well as being a major fuel for colonocytes.

\section{b) Nutritive function}

The intestinal microflora can markedly enhance the nutritional status of the human body through the manufacture of B-vitamins such as biotin, B3, B6 and folic acid. In addition the gut microflora ha also an important complementary function in the digestion of dietary components such as plant polymers(8)

\section{c) Immunological function}

There is accumulating evidence that various lactic acid bacteria cell components directly stimulate the immune response of the host(9). In some cases a synergistic and adjuvant effect has been noted representing a general enhancement of the immune status of the individual as a result of probiotic dosage. Such a general enhancement may also assist the host in suppressing tumours as indicate by available evidence from extensive animal studies(10).

The gastrointestinal tract is an important organ in the defence of the host against harmful organisms and various metabolites including carcinogens.

The indigenous microflora, or their antigens, can penetrate the epithelial barrier of the intestine and stimulate and modulate the activity of the immunocompetent cells. Immuno-competent cells have been suggested to undergo activation by the microflora in the gut and transported to other mucosal sites such as the respiratory and the urogenital tract. The intestinal microflora, or their antigens, can penetrate the epithelial barrier of the intestine, thus stimulating the immunocompetent cells(11). This action may involve the modulation of monocyte-macrophage phagocytic and killing activities, and a modulation on the production of M-CSF, IFNs (gamma, alpha and beta) and interlukins IL1, IL-2, and IL-6(12).

\section{d) Protective function}

The protective action of the beneficial intestinal microflora and probiotic organisms largely depends on two basic properties:

\section{i) Anti-microbial activity}

The anti-microbial activity of lactic acid bacteria is mediated by the production of lactic, acetic and other organic acids, diacetyl, hydrogen peroxide and bacteriocins (at least 14 bacteriocins have been fully described)(13). These anti-microbial agents suppress the multiplication of pathogenic and putrefying bacteria. Several bacteriocins produced by probiotic bacteria have been reported. Some examples include acidophilin, lactobacillin and lactodin known to display anti-microbial activity against E. Coli, Salmonella and Shigella. Recently, 
Dr Ohhira from Japan described the isolation and characterisation of a bacteriocin isolated from $\mathrm{E}$. faecalis TH-10 found to inhibit the MRSA causing Staphylococcus aureus bacteria(14).

A recent summary of 22 clinical human trials designed to test the effectiveness of probiotics in the treatment of diarrhoea showed that in all the studies except in one ( 38 patients out of a total of 3,546 patients) a significant reduction and/or prevention of diarrhea was recorded. The most significant difficulty in the trial was reported to the stability and reliability of the Probiotic bacteria and preparations used(15).

\section{ii) Antimutagenic and anticarcinogenic activity}

The mechanisms of antimutagenicity of the lactic acid bacteria (LAB) remain speculative; however, the antimutagenic activity of products fermented by LAB has been well established in vitro against a large spectrum of mutagens(16). This effect has been detected against a range of mutagens and promutagens in various test systems based on microbial and mammalian cells. Remarkably, recent reports suggest that short chain fatty acids have potential effects at the DNA level in terms of rectifying genetic misreading(17).

Since most of the probiotic bacteria produce various types of short chain fatty acids (SCFA), it can be speculated that these SCFA may be responsible for antimutagenic effects. Butyrate for example has been shown to inhibit the synthesis of DNA of cancer cells and to suppress cancer-specific properties of the cells, which recover normal molecular and cellular characteristics(17).

It has also been suggested that the anti-mutagenic and anti-carcinogenic properties of the lactic acid bacteria may be due to a lowering in faecal enzymes involved in the conversion of pro-carcinogens to carcinogens. Such probiotic bacteria also lower levels of harmful enzymes such as B-glucosidase and B-glucoronidase responsible for the production of harmful nitrosamines.

Another possible mechanisms has been suggested involving the irreversible binding and neutralisation of the mutagen by the probiotic bacteria(18).

\section{DESIRABLE PROPERTIES OF PROBIOTIC BACTERIA}

There is growing consensus that the intestinal flora is closely related to the host's health and proneness to disease. The predominance of harmful bacteria in the intestine may ultimately lead to various disorders, while useful bacteria act by inhibiting or eliminating their harmful counterpart and exerting a plethora of physiological functions. These beneficial effects are beyond question. The many claims that have been made include suppression of diarrhoea, relief of lactose intolerance, neutralisation of mutagens and carcinogens and stimulation of mucosal and systemic immunity. However, some of the reported results show variability which relate to the type of micro-organisms in the probiotic, the method of production and administration and the viability of the probiotic preparation. It is a wellknown fact that commercially available probiotic preparations display wide variations in $\mathrm{LAB}$ counts. In some cases LAB strains other than the ones listed are found in the preparation. In extreme case some preparations have been found to contain no viable $\mathrm{LAB}(19)$. A recent British study found that only 2 out of 13 commercially available pro-biotic preparations tested in the U.K. contained the stated type and potency of bacteria(20).

Some of the desirable properties of probiotic bacteria and supplements are described below:

\section{Stability and technical properties}

- Ability to maintain verified viability

- Good acidity profile

- Maintenance of colonising properties throughout processing and storage

- Accurate strain identification and combination

- Stability and no molecular and/or structural damage following preparation method.

\section{Health and clinical properties}

- Resistance to acid and bile

- Adherence to human intestinal cells

- Colonisation of the Human gut

- Production of organic acids and anti-microbial substances

- Pro-biotic strains with validated health effects

It is essential that the various strains of bacteria present in the probiotic preparation are able to survive the location where it is presumed to be active and proliferate and colonise at this specific location. The most significant attribute of the microorganisms is their ability to produce high levels of organic acids and acidity profile critical to their ability to counteract the presence of harmful and pathogenic micro-organisms. Unless this is achieved, the subsequent expected benefits of the probiotic bacteria will never eventuate.

\section{PROBIOTICS SUPPLEMENTS COMPARISON}

The crucial feature of a probiotic is that it should contain living micro-organisms, however, production methods, processing and storage conditions may adversely affect viability. The most commonly used forms of presentation are liquid/slurry and freeze-dried products. As discussed earlier, many commercial preparations fall short in delivering viable preparations of suitable micro-organisms. This very fact has been the Achilles heel of the probiotic industry unable to overcome the limited self-life and poor survival rates of bacteria in probiotic preparations.

Following many years of intensive investigations a successful alternative to the traditional methods of delivery have been developed by a Japanese research team(21). It involves an encapsulated fermented paste containing 12 strains of LAB. This innovative approach represents a breakthrough in Pro-biotic technology and delivery; the bacteria are fully viable and functional, including 12 strains from four major groups of LAB. The acidity profile of this preparation is excellent, capable of producing organic acids at a reported rate 6.6 greater than 
Table 1: Comparisons of different types of lactic acid bacteria supplements

\begin{tabular}{|c|c|c|}
\hline Liquid preparations & Freeze dried powder & Fermented paste capsules* \\
\hline $\begin{array}{l}\text { Short self-life } \\
\text { Bacteria damaged by } \\
\text { Pasteurization and Centrifugation } \\
\text { Additives and preservatives may } \\
\text { be used. } \\
\text { Transport and storage is difficult } \\
\text { Normally only one or two strains } \\
\text { are used } \\
\text { Damaged by stomach acidity }\end{array}$ & $\begin{array}{l}\text { Severe damage by freeze- drying } \\
\text { Powder shelf-life is short } \\
\text { Powder absorbs water, bacteria } \\
\text { become activated and die away } \\
\text { Poor adherence, colonization and } \\
\text { survival in the gut } \\
\text { Addition of stabilizers and } \\
\text { preservatives }\end{array}$ & $\begin{array}{l}\text { Enteric coated capsule ensures } \\
\text { survival of bacteria past the } \\
\text { acidity of the stomach } \\
100 \% \text { chemical, additive and } \\
\text { preservative free making it ideal } \\
\text { for those suffering with chemical } \\
\text { and food allergies. } \\
\text { No damage to bacterial cell walls } \\
\text { No centrifugation, freeze drying } \\
\text { or harmful chemical methods } \\
\text { Long shelf life. Up to } 1 \text { year at } \\
\text { room temperature and no less } \\
\text { than } 2 \text { years at } 4 \text { oC. } \\
\text { Storage and transportation is easy } \\
\text { Refrigeration is not essential, } \\
\text { however, it is recommended }\end{array}$ \\
\hline
\end{tabular}

other beneficial bacteria(22). In addition, it contains a particular strain, E. faecalis TH10, with demonstrated in vitro effectiveness against, MRSA (Staphylococcus aureus)(14), H. pylori (causative agent in stomach ulcers)(23) and E. coli 0157 ( food poisoning agent). A direct comparison of lactic acid bacteria supplements are presented in Table 1 .

\section{SUMMARY AND CONCLUSIONS}

There is growing consensus and evidence which indicates that the intestinal microflora is a decisive factor which regulates and maintain the health of the host and its ability to overcome disease. Emerging evidence suggests that, among other benefits, the beneficial intestinal bacteria have a key role in the nutritional status of the host, neutralization and elimination of mutagens and carcinogens, stimulation of the immune system, and competition and elimination of harmful organisms. A key feature of the useful probiotic bacteria is their ability to produce significant levels of organic acids and establishing an acidic environment in the intestinal system as a prerequisite for further colonisation and the mediation of further health benefits to the host. A number of factors can change the balance in favour of harmful bacteria. The presence of high levels of harmful organisms in the intestine will increase the production of putrefactive substances, toxins and carcinogens and ultimately lead to various disorders.

Maintenance of a satisfactory balance of the intestinal flora is desirable and could be achieved by oral bacterio-therapy and the use of probiotic supplements. Many available commercial preparations fall short in delivering viable preparations of suitable micro-organisms with current production methods, processing and storage conditions adversely affecting their viability, quality and effectiveness. A recent innovation, of a fermented paste probiotic*, represents a brea kthrough in probiotic technology and delivery.

\section{REFERENCES:}

1. O'Sullivan, M., et al., J. Probiotics, 1992. Dec. Vol. 3.

2. Mitsuoka, T., Asia Pacific J. Clin Nutr, 1996, 5:20-24.

3. The First Australian Symposium on intestinal flora and human health. Special Supplement to the Asia Pacific Journal of Clinical Nutrition, 1996, Volume 5, No. 1, 1-56.

4. 4.Gilliland SE., Walker DK. J. Dairy Sci. 1990; 73:905-911.

5. 5.Gilliland SE.App. Environm. Microbiol. 1977; 33: 15-18.

6. 6.Conway, PL., Asia Pacific J Clin Nutr. 1996 5:1014.

7. Topping DL. Asia Pacific J Clin Nutr. 1996 5:15-19.

8. Hobson, P.N. and Wallace, R.J. PartI and PartII. CRC Critical Reviews in Microbiology, 1982, 9, 165-225(I) and 253-320(II).

9. Havenaar, R, Spanhaak, S. Current Biology. 1994; 5:320-325.

10. Adachi S.. The Lactic Acid bacteria in health and disease, 1992, vol.1, 233-261

11. Havenaar, R. and Huis In't Veld, J.H.J., The Lactic Acid bacteria in health and disease, 1996, vol.1, 151170.

12. Famularo, G., Probiotics 2., Chapman and Hall, 1997,133-161.

13. Klaenhammer, T.R., 1988, Biochimie, 70, 337-379.

14. Ohhira, I., et al. Japanese Journal of Dairy and Food Science, 1996, Vol. 45, No. 4, 93-96.

15. 15. Gary Elmer et al., JAMA 1996, vol275(11).

16. Lankaputhra, W.E.V. and Shah, N.P. 1998, Mutation Research, 397, 169-182.

*OMX capsules 
17. Tanaka,Y. Arch. Biochem. Piophysics, 1990, 276, 415-423.

18. Orrhage, K., et al., Mutation Research,1994, 311, 239248.

19. Gilliland, S.E., 1981, Okla. Agric. Exp. Sta., 108, 6163.

20. Hamilton-Miller, et.al.,Br. Med. J., 1996, 312, 55-56.
21. Ohhira, I. And Nakae, T. Japanese Journal of Dairy and Food Science, 1987, Vol. 36, No. 2, 69-75.

22. Ohhira, I. A Review. Japanese Journal of Dairy and Food Science, 1992, Vol. 41, No. 3, 97-98.

23. C. Mishra and Lambert, J. Festival of Microbes, Ausralian Society for Microbiology, Scientific Meeting and Exhibition, 1997, P07. 22. 